\title{
Schistosoma mansoni no Maranhão entre 1997 e 2019: uma prospecção tecnológica e científica
}

\author{
Schistosoma mansoni in Maranhão between 1997 and 2019: a \\ technological and scientific prospecting
}

\author{
Cleiane Dias Lima ${ }^{1}$ \\ Dallyla Souza de Andrade ${ }^{1}$ \\ Gleidiany da Costa Moreira ${ }^{1}$ \\ Clanecy Alves Soares Lima² \\ Ângelo Afonso Ferreira Sousa ${ }^{1}$ \\ Raimundo Leilton Santos Sousa ${ }^{1}$ \\ Antonia de Sousa Leal ${ }^{1}$ \\ Nayra da Costa Silva Rêgo ${ }^{3}$ \\ Jefferson Almeida Rocha ${ }^{4}$ \\ ${ }^{1}$ Universidade Federal do Maranhão, Grajaú, MA, Brasil \\ ${ }^{2}$ Faculdade Santa Terezinha, CEST, São Luís, MA, Brasil \\ ${ }^{3}$ Universidade Estadual do Piaú, Teresina, PI, Brasil \\ ${ }^{4}$ Universidade Federal do Maranhão, São Bernardo, MA, Brasil
}

\begin{abstract}
Resumo
A esquistossomose é uma doença parasitária que tem o homem como hospedeiro definitivo e comum em locais sem saneamento básico. Sendo assim, esta pesquisa tem o objetivo de realizar uma prospecção científica e tecnológica sobre a esquistossomose no Estado do Maranhão. A prospecção tecnológica foi realizada por meio da busca nas bases: EPO, USPTO, INPI e DII, e por busca em artigos científicos nas bases: Scielo, Bireme, PubMed, Web of Science, Scopus e Science Direct, de 1997 até 2019. Os resultados para patentes mostraram que a base USPTO obteve o maior número de publicações, com 1.879 registros. Nos resultados para prospecção científica, observa-se que para o descritor "Schistosoma mansoni" a base Bireme lidera com 8.063 e para o "Schistosoma mansoni" and "Maranhão" diminui para 21 artigos. Dessa forma, percebe-se que é necessário mais incentivo e investimentos para a realização de mais pesquisas relacionadas à esquistossomose, isso devido ao seu impacto na saúde pública e os poucos tratamentos quimioterápicos encontrados.
\end{abstract}

Palavras-chave: Helminto. Esquistossomose. Doença Parasitária.

\begin{abstract}
Schistosomiasis is a parasitic disease, which has man as a definitive and common host in places without basic sanitation. This research aimed to carry out a scientific and technological research on schistosomiasis in the state of Maranhão. The technological prospection was carried out through the search in the bases: EPO, USPTO, INPI and DII, and the search for scientific articles in the databases: Scielo, Bireme, PubMed, Web of Science, Scopus and Science Direct from 1997 to 2019. The results for patents showed that the USPTO base obtained the highest number of publications with 1.879 records. In the results for scientific prospection, it is observed that for the descriptor "Schistosoma mansoni" the Bireme base leads with 8,063 and "Schistosoma mansoni" and "Maranhão" decreases to 21 articles. Thus, it is perceived that more incentive and investments are needed to conduct more research related to schistosomiasis, impact on public health and the few chemotherapy treatments found.
\end{abstract}

Keywords: Helminth. Schistosomiasis. Parasitic disease.

Área Tecnológica: Prospecção Tecnológica. 


\section{Introdução}

A esquistossomose continua sendo um problema de saúde pública em várias partes do mundo, principalmente na África, onde vivem $92 \%$ de todas as pessoas que necessitam de quimioterapia preventiva para esquistossomose. Dos 78 países considerados endêmicos para a esquistossomose, apenas 52 países têm populações que necessitam de quimioterapia preventiva. O número total de pessoas que precisam de quimioterapia preventiva em todo o mundo em 2018 foi de 229,2 milhões, dos quais, 124,4 milhões eram crianças em idade escolar (WHO, 2018).

$\mathrm{O}$ impacto da doença está vinculado à mortalidade e à morbidade, tornando-a uma das mais importantes infecções humanas causadas por helmintos, sendo uma situação de preocupação mundial, já que, em se tratando de repercussão e importância, ocupa o segundo lugar no mundo, ficando atrás apenas da Malária, que afeta cerca de 200 milhões de pessoas e que 779 milhões estão em risco de infecção, resultando em 280 mil mortes por ano (MORAES, 2012; NASCIMENTO et al., 2013; WHO, 2018.

A esquistossomose é uma doença parasitária comum em comunidades rurais de baixa renda e ocorre em mais de 70 países, afetando mais de 200 milhões de pessoas como já mencionado. Esse é considerado um gravíssimo problema de saúde pública mundial (MORAES et al., 2011). Estima-se que cerca de 25 milhões de pessoas vivem em áreas sob o risco de contrair a doença. No Brasil, os estados das Regiões Nordeste e Sudeste são os mais afetados, sendo que a ocorrência está diretamente ligada à presença dos moluscos transmissores (BRASIL, 2015).

No Maranhão, a esquistossomose é encontrada, predominantemente, nas planícies ocidentais, que são uma das regiões mais pobres do estado (MENDES et al., 2017). Essa microrregião possui vegetações formadas por imensos campos alagados, o que facilita a incorporação de atividades rotineiras. Assim, a esquistossomose é comum nessa região, uma vez que as pessoas que vivem lá têm várias razões para manter contato com a água contaminada (FERREIRA et al., 1998).

Sobre a classificação biológica, os parasitas helmintos do gênero Schistosoma pertencem ao grupo Platyhelminthes, à classe Trematoda e à subclasse Digenea. Os trematódeos digenéticos são organismos integrados adaptados ao parasitismo, possui um complexo ciclo biológico, compreendendo hospedeiros intermediários e definitivos. Muitas espécies de trematódeos afetam a população humana em regiões tropicais e subtropicais do mundo, particularmente nas áreas menos desenvolvidas. As esquistossomoses estão entre as doenças de mais alta endemicidade do planeta; elas são devidas a helmintos trematódeos do gênero Schistosoma (CHITSULO et al., 2000; STEINMANN et al., 2006; MORAES et al., 2011).

Existem outras espécies do gênero Schistosoma que também causam a esquistossomose: S. intercalatum, S. mekongi, S. malayensis, S. hematobium, S. japonicum e S. mansoni. Os principais agentes etiológicos com grande relevância na medicina humana são: $S$. haematobium, $S$. japonicume, S. mansoni, estes são vermes achatados da classe dos trematódeos, cuja família é a Schistosomatidae (CANTANHEDE; FERREIRA; MATTOS, 2011; BRASIL, 2010).

No que diz respeito ao $S$. mansoni, este possui ciclo biológico bem complexo, pois passa por diversas fases e vive em hospedeiros e em ambientes diversificados com uma grande capacidade de adaptação, multiplicação e resistência. As fases do ciclo biológico do $S$. mansoni compreendem dois períodos de vida parasitária: um no hospedeiro intermediário e outro no hospedeiro 
definitivo. Entre as fases parasitárias há dois períodos de vida livre em meio aquático: as fases de miracídio e cercaria (ROSS et al., 2002; BLANCHARD, 2004; GRYSEELS et al., 2006). S. mansoni, em geral, realizam ciclo assexuado nos moluscos do gênero Biomphalaria, enquanto que os espécimes adultos são encontrados nos vasos mesentéricos e no sistema porta de mamíferos.

Quanto aos vermes adultos de S. mansoni, eles se alojam aos pares em vênulas terminais do plexo mesentérico inferior que drenam a parede intestinal. A cada dia, cerca de 300 ovos, medindo $110-180 \mu \mathrm{m}$ por $45-70 \mu \mathrm{m}$, são eliminados pela fêmea no interior das vênulas. $\mathrm{Na}$ inflamação, a presença dos ovos no hospedeiro pode resultar em ruptura da parede da vênula, liberando os ovos nos tecidos perivasculares e, finalmente, na luz intestinal. Cinco anos é a vida média do $S$. mansoni, embora alguns casais de parasitas possam viver mais de 30 anos eliminando ovos (ROSS et al., 2002; GRYSEELS et al., 2006).

O ciclo se inicia com a contaminação do ambiente hídrico, por meio de fezes contaminadas com ovos de $S$. mansoni, que eclodem e liberam larvas ciliadas (miracídios) que penetram nos caramujos. No interior dos moluscos, estes se multiplicam e se transformam em larvas denominados cercárias. Quando livres na água, as cercárias constituem-se em formas infectantes para o homem, que, tendo contato com a água contaminada, propicia a penetração via cutânea. Ao penetrar na pele humana, as cercárias se transformam em esquistossômulo e, por meio da circulação sistêmica, migram até os pulmões e o fígado. Em cerca de cinco semanas após a infecção, já estando alojados nas veias mesentéricas, os vermes atingem a maturidade sexual e se inicia a postura de ovos (GUIMARÃES, 2007; ROSS et al., 2002; BLANCHARD, 2004; GRYSEELS et al., 2006).

O grande desafio para a eliminação do parasita descrito no ciclo acima é o tratamento quimioterápico eficaz. Um dos primeiros fármacos utilizados no tratamento da esquistossomose foi a oxamniquine que apresentou efeitos principalmente contra a espécie $S$. mansoni. No entanto, devido à presença de efeitos adversos, não se obteve sucesso no tratamento, ocasionado o seu abandono (PICA-MATTOCCIA; CIOLI, 1983; BRASIL, 2014)

A quimioterapia sistemática tem sido por muito tempo uma estratégia no tratamento da esquistossomose. Assim, em 1970, o Praziquantel (PZQ) começou a ser desenvolvido para esse fim, sendo usado até os dias atuais (WEBBE; JAMES, 1977). Porém, sabe-se agora que alguns organismos desenvolveram resistência ao PZQ, além disso, ele não tem propriedades profiláticas, sendo assim, torna-se ineficaz contra estádios larvais do parasita (MORAES, 2011; COUTO et al., 2005; BRASIL, 2014).

Diante do que foi exposto, este estudo prospectivo por meio de métodos qualitativo e quantitativo tem como finalidade conhecer a atual realidade das pesquisas já realizadas e das tecnologias produzidas no estado do Maranhão contra essa doença negligenciada, de ampla distribuição global, e da importância da informação para o seu monitoramento e controle. Portanto, neste estudo, pretende-se agregar valores às informações do presente, transformando-as em conhecimento, subsidiando estratégias para as futuras pesquisas.

\section{Metodologia}

A metodologia da pesquisa trata-se de uma prospecção científica e tecnológica desenvolvida pela busca de trabalhos científicos e patentes sobre a esquistossomose no Maranhão, a fim de 
verificar o processo e os avanços tecnológicos e diagnosticar a atual realidade, trazendo novos avanços terapêuticos relacionados ao tema discutido. Para prospecção tecnológica foram utilizados os bancos de dados: European Patent Office (EPO), United States Patentand Trademark Office (USPTO), Derwent Innovations Index (DII) e Instituto Nacional da Propriedade Industrial (INPI), utilizando-se como descritores as palavras-chave "Schistosoma mansoni" e "Schistosoma mansoni" and "Maranhão".

A prospecção cientifica se deu pela busca de artigos, utilizando as bases de dados: Scientific Electronic Library Online (Scielo), Portal Regional da Biblioteca Virtual em Saúde (Bireme), PubMed, Thomson Reuters (Web of Science), grupo Elsevier group (Scopus) e Science Direct. Os descritores utilizados na busca foram: "Schistosoma mansoni", "Schistosoma mansoni" and "Maranhão", considerando todos os documentos que apresentaram esse termo no título e/ou resumo.

Além dessas palavras-chave, o trabalho contou com a presença de alguns recursos de pesquisa, como as aspas, para que pudessem ajudar a encontrar o maior número possível de documentos. Foram analisados os pedidos de patentes depositados e os trabalhos científicos publicados nos últimos 22 anos no período de 1997 a 2019. Com base em todos os resultados das buscas foi realizada uma análise comparativa entre as bases de dados, a fim de avaliar como vêm se desenvolvendo as pesquisas e as publicações sobre a esquistossomose no Maranhão nesses últimos anos, para, a partir desses resultados, poder intervir com novas estratégias terapêuticas.

Os resultados obtidos pela pesquisa realizada nas bases de dados citadas foram organizados em forma de tabelas e de gráficos, no sentido de melhor explorar as informações fornecidas por cada base de dados.

\section{Resultados e Discussão}

Os resultados obtidos por meio da pesquisa realizada nas bases de dados citadas foram organizados em forma de tabelas e gráficos para, assim, melhor explorar as informações fornecidas por cada base de dados.

\subsubsection{Prospecção Tecnológica}

Ao analisar as patentes depositadas nas bases, utilizando o descritor "S. mansoni" até o ano de 2019, foi possível observar um total de 21 pedidos de patentes, já para o descritor "S. mansoni" and "Maranhão" não foi encontrado nenhuma patente. No Brasil, nos últimos 22 anos foram registradas um total de 18 patentes no INPI (1\%), sendo que por ano a quantidade máxima registrada foram quatro patentes. A maioria dos pedidos foi registrada na base americana USPTO com 1.879 (87\%), seguido pela base europeia DII com $211(10 \%)$ e depois a EPO com $47(2 \%)$ patentes (Figura 1$)$. 
Figura 1 - Número de patentes por ano sobre Schistosoma mansoni entre 1997 e 2019

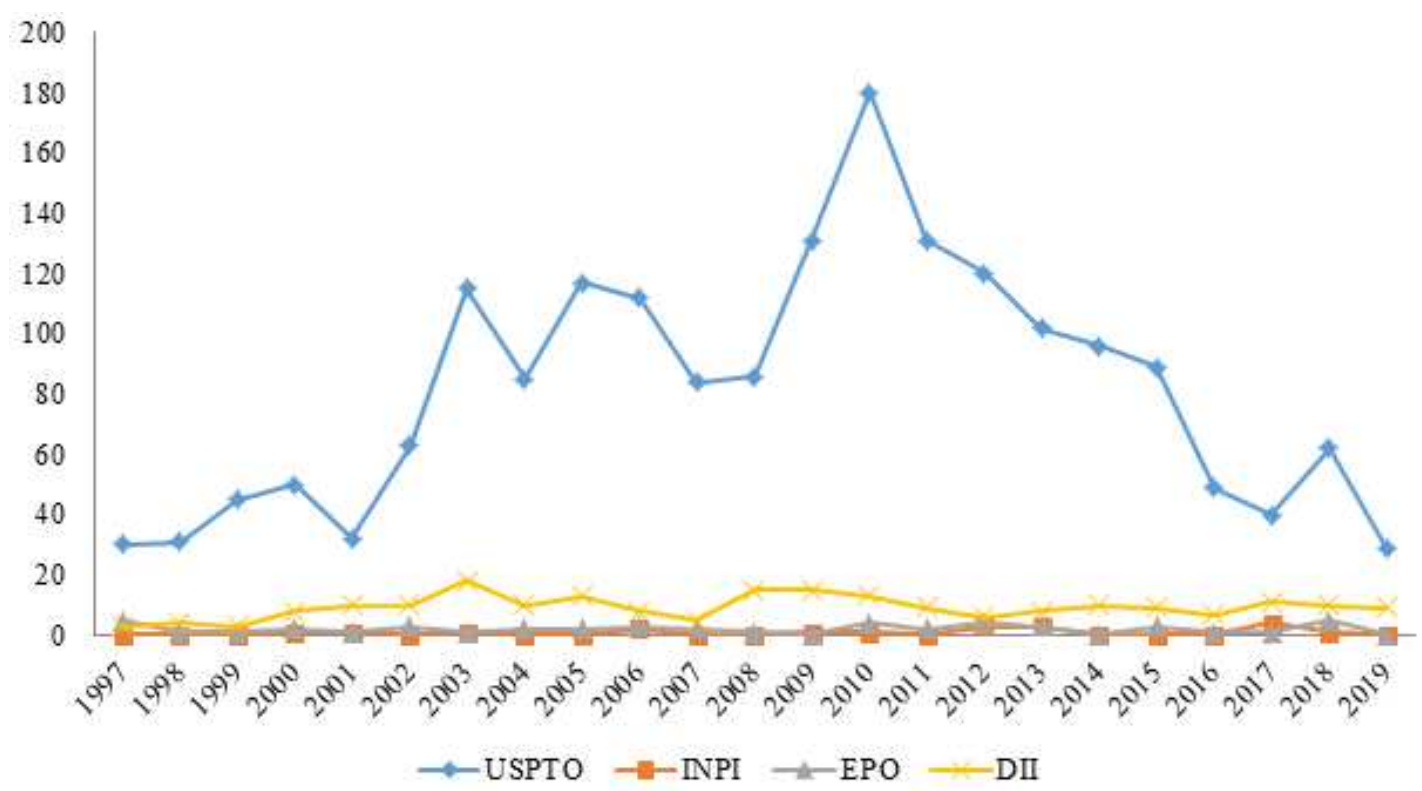

Fonte: Elaborada pelos autores deste artigo (2019)

Ao observar esses dados, percebe-se que a base de dados brasileira (INPI) apresenta um número baixo de pedidos de patentes referentes à $S$. mansoni, a menor entre todas as analisadas. Esse resultado pode ser reflexo da falta de incentivo por parte de políticas públicas, como também pelo baixo investimento de empresas públicas e privadas (IPEA, 2017).

Além disso, a base do INPI requer um extenso período para a tramitação do registro de uma patente, o que também contribui negativamente, levando pesquisadores e empresas a buscarem outras bases com depósito mais rápido (SOUZA, 2015). Já as bases internacionais, em especial a USPTO e a DII, lideram em número de patentes publicadas.

Desde 1997 já foi possível encontrar patentes sobre a esquistossomose, tanto na base nacional quanto nas internacionais, sendo que no final da década de 1990 observou-se um crescimento de publicações em todas as bases. Esse aumento pode ser explicado pelo fato de nesse período a Organização Mundial de Saúde (OMS) ter dado ênfase ao tratamento quimioterápico e ao controle da morbidade, o que possibilitou as novas pesquisas e fez surgir as patentes (WHO, 2010).

Na base americana (USPTO), esse número tende a aumentar com o passar dos anos com algumas oscilações, esse aumento pode estar relacionado à nova estratégia de controle da doença, sendo esse controle conjugado da esquistossomose ao das geo-helmintoses, que foi dirigido para grupos de alto risco sem diagnóstico individual prévio (WHO, 2010).

Para o descritor "Schistosoma. mansoni" and Maranhão, não foi encontrada patente alguma nos bancos de dados, por isso, acredita-se que seria de grande valia que houvesse mais investimentos em pesquisas, pois a esquistossomose constitui um relevante problema de saúde pública no Maranhão, que tem mostrado, entre os estados nordestinos, altos índices de casos nos últimos anos, atingindo 48 dos seus 217 municípios (CANTANHEDE; FERREIRA; MATTOS, 2011). 
A falta de saneamento básico, as irregularidades no abastecimento de água da população e a coleta de lixo inadequada contribuem muito para a contaminação por esquistossomose e também por outras doenças de veiculação hídrica. Além disso, a desigualdade social e o aumento do desemprego permitem que as pessoas vivam em condições precárias, tudo isso contribui para a disseminação de doenças relacionadas às questões socioculturais e ambientais (RAMOS; DA SILVA; SILVA, 2007).

Das 18 patentes encontradas na base do INPI, uma se tratava de uma composição vacinal, compreendendo ao menos um dos sete peptídeos recombinantes altamente reativos aos soros de animais imunizados com as proteínas P22 e P24 de S. mansoni, preferencialmente com micropartículas de quitosana, como carreador e adjuvante imunológico. Os peptídeos propostos na presente invenção induzem resposta imune de células B específica contra a infecção por $S$. mansoni, e a vacina pode ser utilizada para profilaxia da esquistossomose (GÓES et al., 2019).

\subsection{Prospecção Científica}

Para o descritor "S. mansoni" foram encontrados 40.619 artigos publicados nos mais diversos bancos de dados, tanto em periódicos nacionais como nos internacionais, no entanto, quando se restringe à pesquisa, voltando-a para o estado do Maranhão, a quantidade de publicações reduz para 38. Esse resultado está representado na Tabela 1.

Tabela 1 - Número de artigos "Schistosoma mansoni and Maranhão"

\begin{tabular}{ccccccc} 
& Scielo & Bireme & Pubmed & Web of Science & Scopus & Science Direct \\
\hline $\begin{array}{c}\text { "Schistosoma mansoni" } \\
\begin{array}{c}\text { "Schistosoma mansoni } \\
\text { and Maranhão" }\end{array}\end{array}$ & 685 & 8.063 & 6.723 & 8.965 & 8182 & 8.001 \\
\hline
\end{tabular}

Fonte: Elaborada pelos autores deste artigo (2020)

A base Bireme que é nacional apresentou a terceira maior quantidade de artigos publicados, 8.063, para o tema pesquisado, representando $19 \%$ das publicações (Tabela 1). Sabe-se que o Brasil está entre os países que mais produzem pesquisasno mundo, ocupando o $13^{\circ}$ lugar em acesso aberto (GUIMARÃES, 2018).

Para o descritor "S. mansoni" and "Maranhão", a base de dados Bireme também obteve maior número de publicações, 11 artigos (Tabela 1). Observa-se que existem poucos artigos publicados no estado do Maranhão,mesmo com a evidência de uma grande prevalência da esquistossomose nesse estado. Essa doença constitui um problema de saúde pública desde 1920 no Maranhão (NASCIMENTO, 2011).

A quantidade de publicações anuais com o termo "S. mansoni" segue uma variação constante nos últimos 22 anos, como está apresentado na Figura 2. Os dados de publicações anuais para o descritor "S. mansoni" mostram que as bases Science Direct, Scopus, Bireme Web of Science e Pubmed apresentaram uma média entre 200 a 450 publicações, no entanto, percebe-se que a base Scielo trouxe resultados bem inferiores aos demais, com aproximadamente 50 publicações por ano (Figura 2). 
Figura 2 - Número de publicações sobre "Schistosoma mansoni" entre os anos de 1997 a 2019

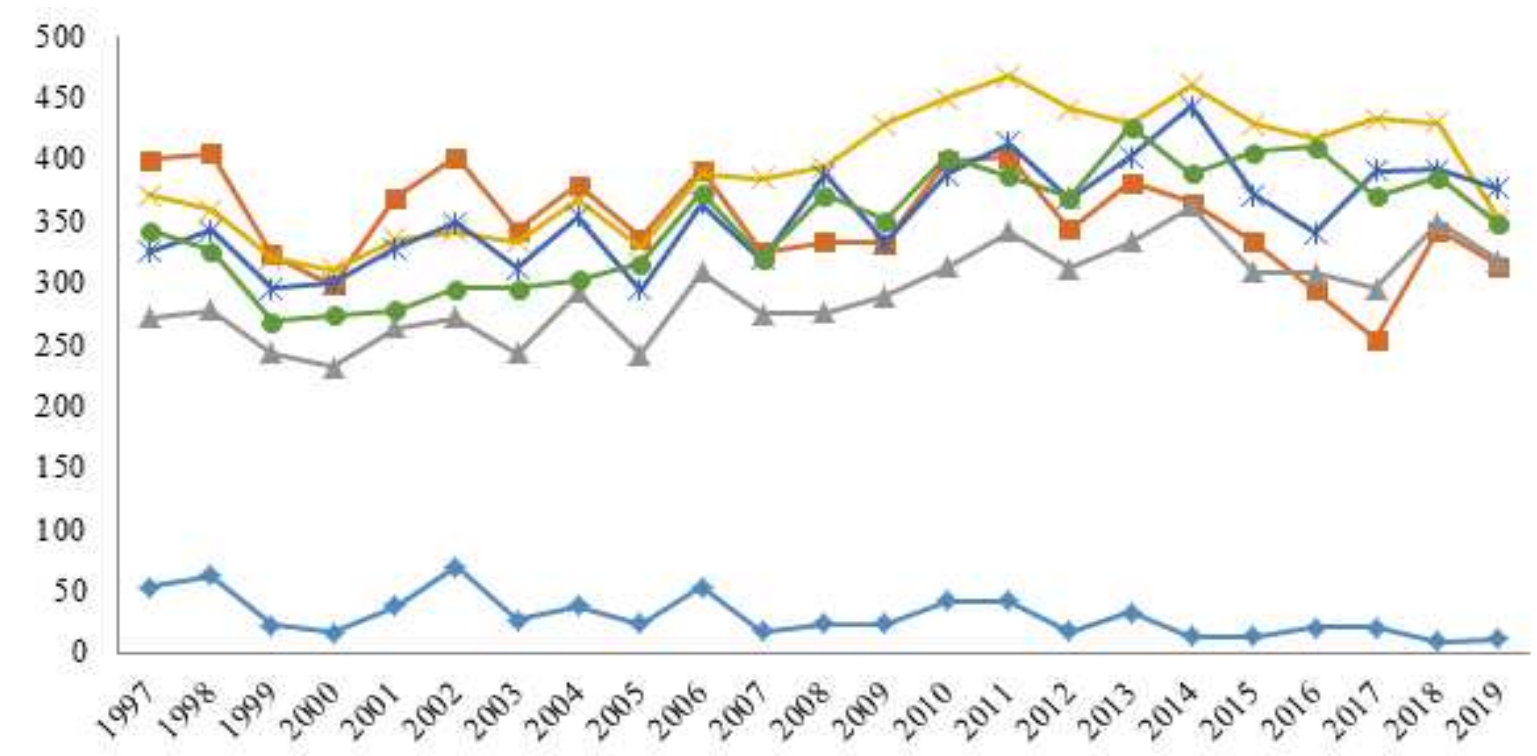

Fonte: Elaborada pelos autores deste artigo (2020)

Em estudo realizado com as folhas de Pilocarpus microphyllus, conhecida popularmente como jaborandi, vários alcaloides dessa espécie foram isolados, a saber, pilosina, epiisopilosina, isopilosina, epiisopiloturina e macaubina. Desses alcaloides estudados, o imidazolo alcaloide epiisopilosina mostrou potente anti-helmíntico in vitro com atividade em baixas concentrações e pode ser considerado um alternativo potencial para o tratamento de infecções causadas por S. mansoni (ROCHA et al., 2017). Esses promissores resultados anti-helmínticos para a epiisopilosina podem abrir novos caminhos para pesquisas nessa área, por exemplo, ensaios in vivo podem ser realizados ou testes contra outras espécies de platelmintos.

Com relação às publicações com os descritores "Schistosoma mansoni" and "Maranhão" nos últimos 22 anos, os dados estão dispostos na Figura 3. Observa-se que o maior número de publicações aconteceu em 2011 com seis artigos, seguido de cinco artigos no ano de 2014 e mais cinco no ano de 2019. Esses resultados se deram a partir de 1997, pois nesse ano o engajamento de periódicos no Brasil tomou impulso e em 2000 foi lançado um movimento internacional do acesso aberto que tornou a produção científica on-line e sem custo, o que pode ter contribuído positivamente para publicações nacionais(MARQUES, 2018).

Figura 3 - Número de publicações sobre "Schistosoma mansoni" and Maranhão entre os anos 1997 a 2019

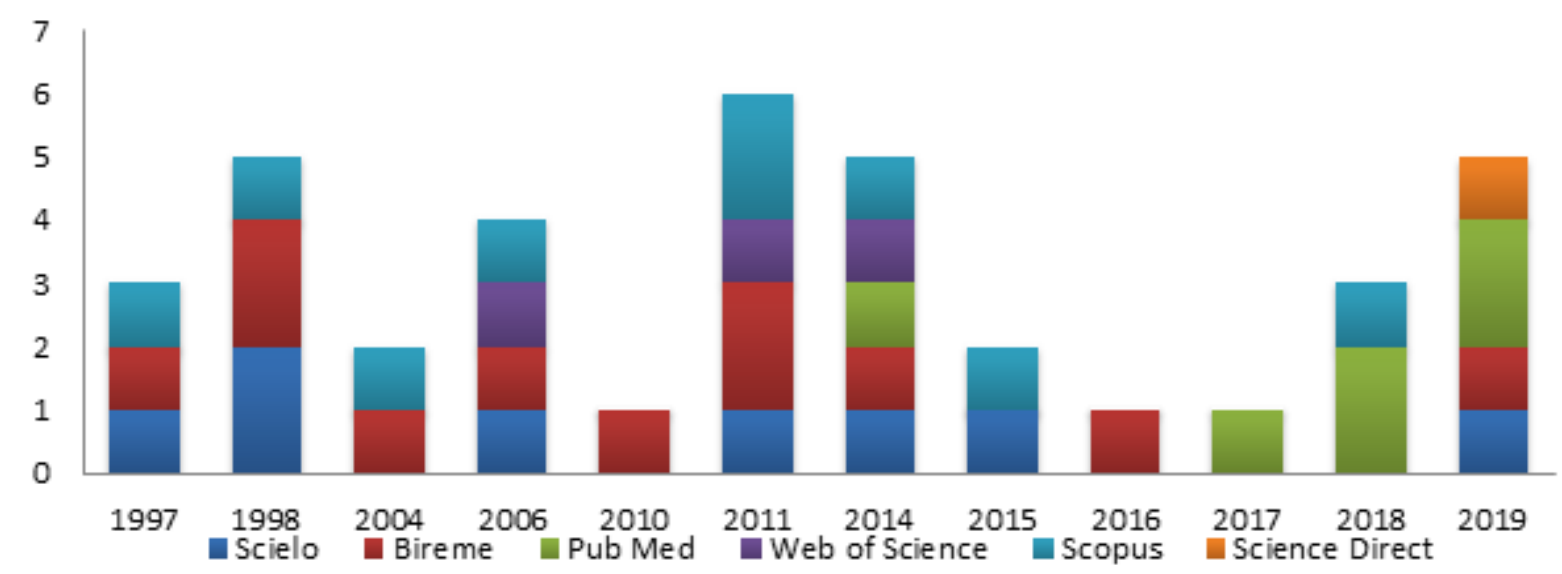

Fonte: Elaborada pelos autores deste artigo (2020) 
Ao observar os 38 artigos na prospecção científica de S. mansoni no estado do Maranhão, nota-se que muitos deles se repetem nas bases de dados e que a quantidade real é apenas de 21 artigos. Entre esses trabalhos que foram encontrados para o Maranhão, a maioria se tratava da epidemiologia da doença (38\%), seguido de estudos da distribuição ou aplicação de extratos contra os caramujostransmissores da esquistossomose (31\%), estudos de caso (13\%), fatores socioeconômicos (6\%) e estudo com S. mansoni (6\%) (CANTANHEDE et al., 2014; SANTOS; MELO, 2011; ROCHA et al., 2018). Ressalta-se que esses estudos estavam bastante relacionados com a Baixada Maranhense, devido são fato de essa ser uma área de maior incidência do hospedeiro intermediário (CANTANHEDE; FERREIRA; MATTOS, 2011). Por se tratar de uma doença que é endêmica nesse estado, não foram identificados estudos sobre atividade anti-helmíntica para o descritor "S. mansoni" and "Maranhão". Isso evidencia uma necessidade pela busca de novas metodologias farmacológicas.

Em um dos artigos analisados com descritor "Schistosoma mansoni" and "Maranhão", que se trata de uma pesquisa realizada recentemente na Planície Ocidental do Maranhão, há evidência de uma grande quantidade de roedores infectados da espécie Holochilus sciureus, a pesquisa mostra que de 225 roedores que foram estudados, 144 animais (64\%) apresentaram ovos de $S$. mansoni em suas fezes, no entanto, essa pode ser mais uma preocupação com a transmissão da doença, já que as fezes infectadas fazem parte do ciclo do verme (SILVA-SOUZA et al., 2019).

Além dessa pesquisa, vários estudos com o mesmo descritor apontaram a prevalência do caramujo na região da Baixada Maranhense devido ao fato de a transmissão dessa parasitose ser costumeiramente em córregos e em riachos que são utilizados pela população para atividades domésticas e para o lazer (ALVIM, 1980; SUCAM, 1990; MARANHÃO, 2002). Além disso, o baixo desenvolvimento socioeconômico e a ausência de ações de educação em saúde e de saneamento são características importantes no quadro de fatores que atuam como determinantes para a transmissão e o estabelecimento dessa parasitose (GAZZINELLI et al., 1998; KATZ et al., 2000).

Apesar do avanço científico e tecnológico que o Brasil vivencia nos últimos anos, com incentivo na formação de doutores, no geral, o número de pesquisadores ainda é baixo para o Nordeste, incluindo o estado do Maranhão (ARAUJO et al., 2016. Além disso, a formação de recursos humanos para trabalhar com pesquisas nesse estado ainda precisa ser incentivada pelos órgãos públicos em virtude da ampliação de estratégias utilizadas no controle de doenças como a esquistossomose.

As informações que foram obtidas com esta pesquisa podem servir como base para novos avanços na ciência, pois, a partir desses dados, é possível observar que ainda existem poucos estudos relacionados ao tratamento dessa parasitose que tanto preocupa o mundo.

\section{Considerações Finais}

De acordo com os dados obtidos por meio da prospecção científica e tecnológica, percebe-se que, com o descritor "Schistosoma mansoni", há algumas pesquisas buscando novos tratamentos e novas metodologias farmacológicas. No entanto, as pesquisas encontradas com o descritor "Schistosoma mansoni" and "Maranhão" apenas fazem uma atualização sobre alguns locais endêmicos, o que não deixa claro o quantitativo por municípios, portanto, percebe-se a necessidade de um estudo epidemiológico no estado do Maranhão, fazendo, assim, um levan- 
tamento de casos dessa parasitose para que sirva tanto como um alerta como um incentivo para a busca de novos estudos no combate a essa doença.

Estudos e pesquisas são fundamentais para enfrentar e até mesmo solucionar problemas que afetam a população nos mais diversos campos. Vários problemas no campo da saúde acometem a população brasileira, sendo que a maioria deles se resolveria com saneamento básico, uma boa alimentação e bom-senso. No entanto, atualmente, as doenças emergentes também têm se tornado um grande desafio, pois germes oportunistas adquirem resistência aos fármacos.

As pesquisas relacionadas à esquistossomose, por exemplo, num contexto mais amplo, como transmissão da doença, controle vetorial, aspectos clínicos e epidemiológicos, estão bem avançadas, porém, quando se especifica um local (Maranhão), a realidade muda bastante, pois os trabalhos se tornam escassos, mesmo havendo registros de caso nesse estado, em que a doença se apresenta de caráter endêmico. Com isso, invenções e patentes precisam ser intensificadas nas pesquisas nacionais, pois isso facilitaria a transferência de tecnologia para a população.

Dessa forma, sabe-se que são necessários mais incentivos e, consequentemente, mais investimentos e financiamentos para que as pesquisas relacionadas à esquistossomose possam ser desenvolvidas, já que esse é um gravíssimo problema de saúde que vem assolando o Brasil e em especial o estado do Maranhão. Além disso, é necessário incorporar e despertar a ciência e a mentalidade científica nos sistemas educacionais e, também, na sociedade em geral, por meio de recursos e de incentivos que tenham como intuito equipar os laboratórios e as universidades.

Enfim, é de grande importância que seja realizado um estudo mais aprofundado sobre esse parasita e, consequentemente, que se encontre uma droga mais eficaz contra essa parasitose, pois o número de publicações relacionadas à esquistossomose a nível mundial demonstra a preocupação e o interesse em erradicá-la globalmente. Por isso, sugere-se a realização de novos estudos e de novas descobertas motivadas e estimuladas pelo setor de ciência e tecnologia do país.

\section{Referências}

ARAUJO, K. S. et al. Prospecção científica e tecnológica da dengue no Brasil e no Maranhão no período de 1994 a 2014. Cadernos de Prospecção, Salvador, v. 9, n. 3, p. 313-322, jul.-set., 2016.

ALVIM, M. de C. A. Esquistossomose no Maranhão. Hiléia Médica, Belém, v. 2, n. 2, p. 151-157, 1980.

BLANCHARD, T. J. Schistosomiasis. Travel Med. Infect. Dis., [s.l.], v. 2, p. 5-11, 2004.

BRASIL. Secretaria de Vigilância em Saúde. Departamento de Vigilância Epidemiológica.

Doenças infecciosas e parasitárias: guia de bolso. 8. ed. rev. Brasília, DF: Ministério da Saúde, 2010.

BRASIL. Relatório do Inquérito de Prevalência de Tracoma em escolares: 2002-2008. Brasília, DF: Ministério da Saúde, 2011.

BRASIL. Secretaria de Vigilância em Saúde. Departamento de Vigilância Epidemiológica. Vigilância da Esquistossomose Mansoni: diretrizes técnicas. 4. ed. Brasília, DF: Ministério da Saúde, 2014. $144 \mathrm{p}$. 
BRASIL. Secretaria de Vigilância em Saúde. Sistema nacional de vigilância em saúde. Relatório de situação - Maranhão: Ministério da Saúde, Secretaria de Vigilância em Saúde. 2. ed. Brasília, DF: Ministério da Saúde, 2015. p. 28.

CANTANHEDE, S. P. D. et al. Freshwater gastropods of the Baixada Maranhense Microregion, an endemic area for schistosomiasis in the State of Maranhão, Brazil: I - qualitative study. Rev. da Soc. Bra. de Medicina Tropical, [s.l.], v. 47, n. 1, p. 79-85, jan-feb, 2014.

CANTANHEDE, S. P. D.; FERREIRA, A. P.; MATTOS, I. E. Esquistossomose mansônica no Estado do Maranhão, Brasil, 1997-2003. Cadernos de Saúde Pública, [s.l.], v. 27, abr. 2011.

COUTO, J. L. A. et al. Esquistossomose mansoni em duas mesorregiões do Estado de Alagoas.

Revista da Sociedade Brasileira de Medicina Tropical, [s.l.], v. 38, p. 301-304, 2005.

CHITSULO, L. et al. The global status of schistosomiasis and its control. Acta Trop., [s.l.], v. 77, p. 41-51, 2000.

FERREIRA L. A. et al. Forma tumoral esquistossomótica encefálica: apresentação de um caso tratado cirurgicamente. Rev. Soc. Bras. Med. Trop., [s.l.], v. 31, n. 1, p. 89-93, 1998.

GAZZINELLI, A. et al. Sociocultural aspects of schistosomiasis mansoni in anendemicarea in Minas Gerais, Brazil. Cad. Saúde Pública, [s.l.], v. 14, p. 841-849, 1998.

GRYSEELS, B. et al. Human schistosomiasis. Lancet, [s.l.], v. 368, p. 1.106-1.118, 2006.

GÓES, A. M. et al. Composição Vacinal com Peptídeos Recombinantes de S. mansoni e uso. Depositante: Universidade Federal de Minas Gerais; Fundação de Amparo à Pesquisa do Estado de Minas Gerais - FAPEMIG. 37F7671463609DCD. Depósito em: 13 de dezembro 2017. Data da Publicação Nacional: 25 de junho de 2019.

GUIMARÃES, M. C. A. Avaliação do Controle e Vigilância do Hospedeiro Intermediário do Schistosoma mansoni, no Vale do Ribeira e observações do seu parasitismo. 2007. $130 \mathrm{f}$. Tese (Doutorado em Saúde Pública) - Faculdade de Saúde Pública, Universidade de São Paulo, São Paulo, 2007.

GUIMARÃES, M. Brasil é o país com mais publicação científica em acesso aberto. Pesquisa Fapesp, São Paulo, ed. 263, 21 jan. 2018. Disponível em: http://revistapesquisa.fapesp.br/2018/01/21/brasile-o-pais-com-mais-publicacao-cientifica-em-acesso-aberto. Acesso em: 30 jan. 2018.

IPEA - INSTITUTO DE PESQUISA ECONÔMICA E APLICADA. Políticas de apoio à inovação tecnológica no Brasil: avanços recentes, limitações e propostas de ações. Organizadores: Lenita Maria Turchi e José Mauro Morais. Brasília: Ipea, 2017. 485 p.: il., gráfs. color. ISBN: 978-85-7811307-0.

KATZ, N.; PEIXOTO, S. V. Análise crítica da estimativa do número de portadores de esquistossomose mansônica no Brasil. Rev. Soc. Bras. Med. Trop., [s.l.], v. 33, p. 303-308, 2000.

LOPES, I. C. et al. Histopathological study of Schistosoma mansoni infection in the murine model using the PC (Pará) and LILA (Maranhão) strains. Rio de Janeiro: Fiocruz, 2011. v. 101.

MARANHÃO. (Estado). Gerência de Qualidade de Vida. Programa de Controle da Esquistossomose. Plano Estadual de Intensificação das Ações de Controle da Esquistossomose (PIACE). São Luís, 2002. 
MARQUES, F. Produção científica acessível. Pesquisa Fapesp, São Paulo, ed. 263, 21 jan. 2018. Disponível em: http://revistapesquisa.fapesp.br/2017/09/22/producao-cientificaacessivel/?cat=politica. Acesso em: 30 jan. 2018.

MENDES, M. F. C. et al. Investigação epidemiológica da esquistossomose mansônica em municípios do Maranhão, Brasil. J. Modelo Geospat, [s.l.], v. 2, n. 3, p. 12-18, 2017. Disponível em: http:// dx.doi.org/10.22615/2526-1746-jgm-2.3-6851. Acesso em: 30 jan. 2018.

MORAES, J. Antischistosomal natural compounds: present challenges for new drug screens. In: RODRIGUEZ-MORALES, A. J. (ed.). Current Topics in Tropical Medicine. Tech: Rijeka, 2012. p. 333-358.

MORAES, J. et al. Evaluation of the in vitro activity of dermaseptin 01, a cationic antimicrobial peptide, Against Schistosoma mansoni. Chemistry \& Biodiversity, [s.l.], v. 8, p. 548-558, 2011.

MORAES, J. Efeito in vitro de extratos e compostos naturais em Schistosoma mansoni. 2011. 236 f. Tese (Doutorado em Biotecnologia) - Instituto de Ciências Biomédicas, Universidade de São Paulo, São Paulo, 2011.

NASCIMENTO, A. I. P. Análise Epidemiológica da Esquistossomose em Áreas de Risco em São Luís - MA. 2011. 112 f. Dissertação (Mestrado Biologia Parasitária) - Centro Universitário do Maranhão, São Luís, 2011.

NASCIMENTO, G. L. et al. Formas Graves da Esquistossomose mansoni: carga epidemiológica e custos no Brasil em 2010. 2013. 73 f. Dissertação (Mestrado em Medicina Tropical) - Faculdade de Medicina, Universidade de Brasília, Brasília, 2013.

PICA-MATTOCCIA, L.; CIOLI, D. Effect of hycanthone administered in vivo unpon the incorporation of radioactive precursors into macromolecules of Schistosoma mansoni. Molecular and Biochemical Parasitology, [s.l.], v. 8, p. 99-107, 1983.

RAMOS, M. C.; DA SILVA, D. C.; SILVA, S. L. C. Educação, saúde e meio ambiente: o caso da esquistossomose no município de itororó-ba. Rev. Saúde Com., [s.l.], v. 3, n. 2, p. 70-76, 2007.

ROCHA, J. A. et al. Computational quantum chemistry, molecular docking, and ADMET predictions of imidazole alkaloids of Pilocarpus microphyllus with schistosomicidal properties. PLoS ONE, [s.l.], v. 13, n. 6, p. e0198476, 2018. Disponível em: https://doi.org/10.1371/journal. pone.0198476. Acesso em: 29 ago. 2019.

ROCHA, J. A. et al. Anthelmintic, antibacterial and cytotoxicity activity of imidazole alkaloids from Pilocarpus microphyllus leaves. Phytotherapy Research, [s.l.], v. 31, n. 4, p. 624-630, 2017.

ROSS A. G. et al. Schistosomiasis. N. Engl. J. Med., [s.l.], v. 346, p. 1.212-1.220, 2002.

SANTOS, A. M.; MELO, A. C. F. L. Prevalência da esquistossomose num povoado do Município de Tutóia, Estado do Maranhão. Revista da Sociedade Brasileira de Medicina Tropical, [s.l.], v. 44, n. 1, p. 97-99, jan-fev., 2011.

SCHELL, S. C. How to know the trematodes. Dubuque: WMC Brown, 1970.

SILVA-SOUZA, N. et al. Parasitological and histological aspects of Holochilus sciureus naturally infected by Schistosoma mansoni. Braz. J. Vet. Parasitol., Jaboticabal, v. 28, n. 4, p. 769-772, Oct.-Dec., 2019. DOI: https://doi.org/10.1590/S1984-29612019075. 
SOUZA, D. Demora na concessão de patentes desestimula a inovação industrial. American Chamber of Commerce for Brazil, [s.l.], 2015. Disponível em: http://www.inova.unicamp.br/ noticia/3580/. Acesso em: 15 jan. 2018.

STEINMANN, P. et al. Schistosomiasis and water resources development: systematic review, metaanalysis, and estimates of people at risk. Lancet Infect. Dis., [s.l.], v. 6, p. 411-425, 2006.

SUCAM. D. R. M. Esquistossomose: comportamento da prevalência em São Luís. São Luís: [s.n.], 1990.

WEBBE, G.; JAMES, C. A comparison of the susceptibility to praziquantel of Schistosoma haematobium, S. japonicum, S. mansoni, S. intercalatum and S. mattheei in hamsters. Z. Parasitenk, [s.l.], v. 52, p. 169-177, 1977.

WHO - WORLD HEALTH ORGANIZATION. Elimination of schistosomiasis from lowtransmission areas: report of a WHO informal consultation. Geneva, atualizado em 2010. Disponível em: http://www.who.int/schistosomiasis/disease/en/. Acesso em: 25 dez. 2017.

WHO - WORLD HEALTH ORGANIZATION. Esquistossomose situação e tendências: dados mundiais. 2018. Disponível em: https://www.who.int/gho/neglected_diseases/schistosomiasis/en/. Acesso em: 14 abr. 2020.

\section{Sobre os Autores}

\section{Cleiane Dias Lima}

E-mail: cleianedias080@mail.com

Graduanda em Ciências Naturais/Química pela Universidade Federal do Maranhão

Endereço profissional: Av. Aurila Maria Santos Barros de Sousa, s/n, Bairro Loteamento Frei Alberto Beretta, Grajaú, MA. CEP: 65940-000.

\section{Dallyla Souza de Andrade}

E-mail: ladydally_andrade@gmail.com

Graduanda em Ciências Naturais/Química pela Universidade Federal do Maranhão.

Endereço profissional: Av. Aurila Maria Santos Barros de Sousa, s/n, Bairro Loteamento Frei Alberto Beretta, Grajaú, MA. CEP: 65940-000.

\section{Gleidiany da Costa Moreira}

E-mail: gleidycosta1@hotmail.com

Especialista em Biodiversidade e Conservação pela UESPI/NEAD em 2017. Técnica em Biologia pela Universidade Federal do Maranhão.

Endereço profissional: Av. Aurila Maria Santos Barros de Sousa, s/n, Bairro Loteamento Frei Alberto Beretta, Grajaú, MA. CEP: 65940-000.

\section{Clanecy Alves Soares Lima}

E-mail: clanecysoares@gmail.com

Bacharel em Enfermagem pela Faculdade Santa Terezinha, São Luís, Maranhão, em 2018.

Endereço profissional: Av. Casemiro Júnior, n. 12 - Anil, São Luís, MA. CEP: 65045-180. 


\section{Ângelo Afonso Ferreira Sousa}

E-mail: angelo.afonso.s@gmail.com

Graduando em Ciências Naturais/Química pela Universidade Federal do Maranhão. Técnico em Química pela Universidade Federal do Maranhão.

Endereço profissional: Av. Aurila Maria Santos Barros de Sousa, s/n, Bairro Loteamento Frei Alberto Beretta, Grajaú, MA. CEP: 65940-000.

\section{Raimundo Leilton Santos Sousa}

E-mail: ssleilton.r@gmail.com

Graduando em Ciências Naturais/Química pela Universidade Federal do Maranhão.

Endereço profissional: Av. Aurila Maria Santos Barros de Sousa, s/n, Bairro Loteamento Frei Alberto Beretta, Grajaú, MA, CEP: 65940-000.

\section{Antonia de Sousa Leal}

E-mail: antonialeal27@gmail.com

Doutora em Biotecnologia pela Rede Nordeste de Biotecnologia, Universidade Federal do Piauí, em 2017.

Endereço profissional: Av. Aurila Maria Santos Barros de Sousa, s/n, Bairro Loteamento Frei Alberto Beretta, Grajaú, MA. CEP: 65940-000.

\section{Nayra da Costa Silva Rêgo}

E-mail: nayralygil@hotmail.com

Doutora em Biotecnologia pela Rede Nordeste de Biotecnologia, Universidade Federal do Piauí.

Endereço profissional: Campus Universitário Ministro Petrônio Portella, Bairro Ininga, Teresina, PI. CEP: 64049-550.

\section{Jefferson Almeida Rocha}

E-mail: jeffersonbiotec@gmail.com

Doutor em Biotecnologia pela Rede Nordeste de Biotecnologia, Universidade Federal do Piauí, em 2018.

Endereço profissional: Campus de São Bernardo, Rua Projetada, s/n, Bairro Planalto, São Bernardo, MA. CEP: 65550-000. 